$0.87 \pm 0.32$ at $90 \%$. This suggests that the integrity of PD reproduction depends on the magnitude of the stimulus that marks its onset. We investigated this relation between magnitude and temporal coding reliability in a recurrent network model. The architecture is constrained by the neuroanatomy of the primate frontostriatal system, and was used to explain primate cortical and subcortical electrophysiology results in sequenceing tasks, and to (accurately) predict human sensitivity to interactions between temporal and spatial coding. Simulations suggest, in agreement with behavioral results, that the encoding of time and motion does not involve entirely separate channels.

Supported by the IFR-Salpêtrière and HFSP-RG71/96B

\title{
Disease and Pathology
}

\section{DP1}

\section{SELECTIVE DEFICITS OF AUDITORY RECOGNITION AND LOCALISATION FOLLOWING CIRCUMSCRIBED HEMISPHERIC LESIONS IN MAN}

S. Clarke*, A. Bellmann, M. Adriani, P. Maeder, R. Meuli, and G. Assal

Division de Neuropsychologie, CHUV, Lausanne; Institut de Physiologie, Faculté de Médecine, Université de Lausanne; and Service de Radiodiagnostic, CHUV, Lausanne, Switzerland

Auditory recognition and auditory spatial functions were studied in patients with circumscribed right or left hemispheric lesions. Semantic recognition of environmental sounds was evaluated in a multiple choice setting comprising 50 sound samples. Auditory localisation and auditory motion perception were tested by simulating different azimuthal positions or the motion of a sound source by varying differences in interaural time (DIT) or differences in interaural intensity (DII); each test comprised 48 to 60 items. Normative data were obtained in over 45 normal subjects. Auditory recognition and auditory spatial functions were found to be disrupted independently in five patients with focal brain lesions. Two patients were excellent in sound recognition but deficient in localisation, whereas the three other patients had difficulties in recognising sounds but localised them correctly. The lesions associated with these deficits involved different parts of the temporo-parietal cortex. Selective deficits in sound recognition were associated with lesions of the temporal pole or the temporal convexity on either the left or the right side. Selective deficits in auditory spatial functions were associated with lesions of distinct parts of the supratemporal plane, the insula and the inferior parietal lobule on either the left or the right side. We propose that auditory information is processed in the human auditory cortex along two distinct pathways, one lateral devoted to auditory recognition and one medial and posterior devoted to auditory spatial functions. 


\title{
DP2
}

\author{
AUDITORY INFORMATION PROCESSING DYSFUNCTION IN A CASE OF VOICE \\ HALLUCINATIONS AND SPASTIC PARAPARESIS

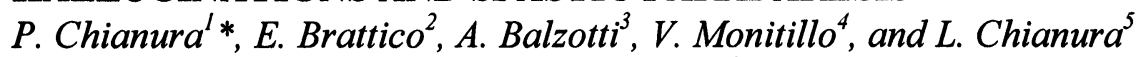 \\ ${ }^{1}$ Department of Mental Health, Bari, Italy, ${ }^{2}$ Cognitive Brain Research Unit, Dept Psychology, Univ \\ Helsinki, Finland, ${ }^{3}$ School of Specialization in Clinical Psychology, Bari, Italy, ${ }^{4}$ Division of Neurology, \\ "S. Maugeri" Foundation, IRCCS, Cassano Murge, Italy, and ${ }^{5}$ Clinical Department of Psychiatry, \\ University Hospital, Bari, Italy
}

Event-related potentials (ERPs) provide a tool for evaluating potential mechanisms underlying neurophysiological information processing dysfunction in schizophrenia. Particularly, a short-duration auditory cognitive ERP component called mismatch negativity (MMN) has been demonstrated to index the accuracy and functionality of the auditory sensory ('echoic') memory system. It is evoked in an auditory oddball paradigm where a sequence of repetitive 'standard' stimuli is interrupted infrequently by a 'deviant' stimulus differing from the standards in one or several physical or abstract parameters. An MMN dysfunction is supposed to be directly related to the degree of sensory memory impairment in schizophrenic subjects (Javitt et al., 1998; Catts et al., 1995; Baldeweg et al., 1998). On the other side, hallucinated voices in schizophrenia have been demonstrated to arise from disrupted speech perception and impaired verbal working memory systems rather than from non-linguistic cognitive or attentional deficits (Hoffman et al., 1999). A 25-years-old patient with spastic paraparesis who after a period of sensory deprivation developed voice hallucinations without other psychotic symptoms, has been studied with ERP method. She was presented with four stimulus conditions: passive/active auditory oddball paradigms with phoneme/complex sound stimuli. Schizophrenics with/without hallucinations and healthy subjects were used as control. Comments are made on the unusual features of the hallucination and on the probable neurophysiological causation as reflected by the ERP measurements.

\section{DP3}

LONG TERM EFFECTS OF CLOSED HEAD INJURY ON NEURAL ACTIVITY ASSOCIATED WITH AUDITORY SELECTIVE ATTENTION

S. Chaudhry-Dijkerman ', M.D. Rugg , D. Gentleman ${ }^{3}$, and J. Gilchrist ${ }^{3}$

${ }^{1}$ School of Psychology, University of St Andrews, St Andrews, Scotland, UK ${ }_{3}^{2}$ Institute of Cognitive Neuroscience, Queen Square, London, UK, and Teaching Hospitals, Ninewells Hospital, Dundee, Scotland, UK

Event-Related Potentials (ERPs) were recorded during a dichotic, three stimulus oddball task.

Compared to closed head injury (CHI) subjects $(n=15)$, the ERPs of control subjects $(n=15)$ showed significantly greater discrimination between attended and unattended frequently occurring stimuli. The lowered discrimination in $\mathrm{CHI}$ subjects resulted from the reduced amplitude of a late 'processing 
negativity'. In addition, whereas the processing negativity observed in the controls was symmetrically distributed, it was asymmetric (left $>$ right) in the patient group. ERPs to infrequently occurring 'target' stimuli (P3b) were also sensitive to attention, but performance and P3b amplitude with respect to attended and unattended targets were comparable in the two groups. Furthermore, ERPs elicited by infrequent 'novel' stimuli (novel P3), also did not differ between the two groups. However, echoing the findings of impaired processing negativity for the frequent stimuli, the early phase of a sustained frontal negativity elicited by unattended targets was also attenuated in the $\mathrm{CHI}$ group. These data suggest that attentional impairment after CHI may be more related to difficulties in the mobilisation of sufficient processing resources than to the inefficiency in inhibiting the processing of distractors, or in directing resources to task-relevant input.

\title{
DP4
}

TEXTURE SEGMENTATION AND VISUAL SEARCH IN PATIENTS WITH FOCAL BRAIN LESIONS

\author{
R. Sireteanu*, B. Krusch-Mielke, B. Dornburg, and R. Rettenbach \\ Max-Planck-Institute for Brain Research, Frankfurt, Germany
}

Texture segmentation and visual search are believed to be part of a so-called "early" visual system, by which primitive visual features can be isolated (c.f Treisman and Gelade, 1980). In previous studies, we have shown that the segmentation of oriented textures and the search for classical visual features is located at a relatively high level in the human visual system. These functions undergo perceptual learning, which transfers from one task to another, from one location in the visual field to another, and between the two eyes of a given subject (Sireteanu and Rettenbach, 1995 and in press). To gain better insight into the possible location of the neural mechanisms involved in these functions, a large number of neurological patients with focal brain lesions of different aetiologies were investigated using stimuli and procedures identical to those of the studies of Sireteanu and Rettenbach. The subjects were 87 patients with focal brain lesions, aged 20-80 years, and 87 control subjects matched for age, gender and socioeconomic status to the experimental subjects. The subjects were recruited either from a rehabilitation clinic $(n=40)$ or from a neurological practice $(n=47)$. The cause of the lesion could be a vascular insult, a ruptured aneurysm, a benign tumour, a cerebral trauma, or a diversity of other factors. The task of subjects was to identify as quickly and correctly as possible the location of an odd target amidst an array of distractors. In the texture segmentation task, the target could be a single discrepant line segment or a group of 16 discrepant line segments in a background of orthogonally oriented lines. In the visual search tasks, the target was a single discrepant item (a plain or a broken circle, a pair of parallel or converging lines, or a single vertical or tilted line) among an array of 3,7 or 15 distractors. To overcome the declining visual capacity with age, the items were large (about 3.5 degrees). Number of errors and response latency were recorded. The test consisted of two sessions, each consisting of four blocks, separated by 1-3 days. The search curves of the patients showed higher intercepts and slopes than those 
of the age-matched control subjects. Performance improved with practice in both groups of subjects. There was no learning overnight; rather, the error rates tended to increase at the beginning of the second testing session. The impairment in performance was age-dependent, with patients in the oldest age group (66-80 years) being most deeply impaired in both tasks. The male patients performed significantly worse than the female patients; this difference was not due to the relatively higher incidence of brain traumata among the male patients. Patients with lesions in the frontal and the right parietal brain showed deeper impairments (higher slopes and higher error rates) in both tasks than patients with other localisations. There were no qualitative differences between the search curves and the learning curves of these groups of patients, neither between the groups of patients and the age- and gender-matched control subjects. Our results confirm studies from our laboratory using the functional Magnetic Resonance Imaging technique, which suggest that visual search tasks activate a complex cortical network, including areas in the frontal and the right parietal cortex (Goebel et al., 1998).

\section{DP5}

VISUAL CONTROL OF BALANCE IN SUBJECTS WITH CONGENITAL NYSTAGMUS M. Guerraz*, J. Shallo-Hoffmann, A. Bronstein, and M. Gresty

MRC Human Movement and Balance Unit, National Hospital for Neurology and Neurosurgery, London, WC1 $3 B G, U K$

The purpose of this study was to investigate the role of vision in the control of balance in patients with congenital nystagmus $(\mathrm{CN}) . \mathrm{CN}$ is an oculomotor disorder characterized by horizontal involuntary eye movements that impair the ability to maintain visual targets on the fovea. In our study, subjects ( patients and 12 controls) were facing a $1.5 \mathrm{~m}$ distant rectangular visual background $(2 \mathrm{~m} \times 3 \mathrm{~m})$ which was stationary or moved $(0.58 \mathrm{~m}$ translation, $10 \mathrm{sec}$ duration) colinearly to the interaural axis. Two visual conditions were manipulated: 1) a background fixation without any foreground and a 2) fixation of a earth-fixed foreground (window frame: $30 \times 24 \mathrm{~cm}$ ), $50 \mathrm{~cm}$ distant from the subject's eyes, placed between the subject and the background. Using a posturography platform and a Shottky camera mounted above the head, the centre of pressure (COP) and the head displacements were measured. Because of the intrinsic visual instability, we hypothesized that $\mathrm{CN}$ subjects may be insensitive to visual cues for the control of posture. Two parameters were measured: 1) COP and head displacements using the average position relative to pre-stimulus position and 2) stability at the level of the COP and the head using the sway path length. Results were as follow: Analysis of body displacement (COP and Head) showed that when the background was moving, body readjustments were of similar amplitude in controls and $\mathrm{CN}$ subjects both in conditions of direct background fixation (body sway in the direction of background motion) and foreground fixation (body sway in the opposite direction of background motion). Analysis of body stability showed that unlike controls, $\mathrm{CN}$ subjects did not benefit from stationary visual cues to maintain upright stance: they were more unstable than controls when stationary cues were available in the visual environment (Stationary foreground). In the absence of any stationary cues (direct fixation of 
the moving background) $\mathrm{CN}$ were as stable as controls. These results showed that $\mathrm{CN}$ subjects were able to pick up visual cues from the visual environment to orient their body in space but not dynamic cues to stabilize their body. These observation are in favor of the hypothesis that $\mathrm{CN}$ subjects have developed the ability to extract visual information during foveation periods (short periods -10 to $100 \mathrm{~ms}$ - when images are most stable on the fovea) but to ignore the smeared visual information during the high-drift velocities that comprise the $\mathrm{CN}$ slow phase.

\section{DP6}

THE BARE BONES OF OBJECT RECOGNITION: IMPLICATIONS FROM A CASE OF APPERCEPTIVE VISUAL AGNOSIA

J. Davidoff * and E. K. Warrington ${ }^{2}$

${ }^{\prime}$ Goldsmiths' College, University of London, UK and ${ }^{2}$ National Hospital, London, UK

Three experiments investigated the performance of a patient (RK) who could name objects when presented in conventional views but showed catastrophic failures in identification from unconventional views. The aim of all three experiments was to assess the properties of the central representations that allow recognition of objects presented in conventional but not unconventional views. All three experiments showed that RK had problems in object identification not apparent from his naming performance. In the first experiment, RK was found to be extremely impaired at recognising the parts of objects even though he could name the whole object. In the second experiment, alterations in colour, shape and parts of objects were undetected in stimuli that he could name. In the third experiment, RK showed considerable difficulty with mirror-images and inversion tasks. The explanation for RK's impaired object recognition could not be attributed to defects to his early visual processing. We argue that RK's recognition is achieved through abstract (object-centred) representations that are global rather than local, and quite independent of their spatial framework. These abstract representations we take to be the essential bare bones for object recognition.

\section{DP7}

OPTIC ATAXIA IS IMPROVED IN CONDITIONS OF 'INDIRECT' POINTING: FURTHER EVIDENCE FOR TWO MODES OF SPATIAL LOCALIZATION

MT. Perenin ${ }^{\prime}$, P. Revol', Y. Paulignan', and A. Vighetto ${ }^{2}$

${ }^{1}$ INSERM Unité 94, 69500 Bron, France, and ${ }^{2}$ Hôpital Neurologique, 69003 Lyon, France

Optic ataxia has been considered as a specific disruption of the visuomotor mechanisms involved in prehension. Although they are grossly inaccurate in reaching and grasping for visual objects, patients with optic ataxia are nevertheless able to discriminate the shape, size and orientation of objects, but also their absolute position (Perenin and Vighetto 1988, Jeannerod et al 1994). Opposite dissociations 
between perception and action observed in both normal and brain-damaged subjects have led to the hypothesis of two separate systems of spatial representations (Milner and Goodale 1995, Perenin 1997). 'Motor', short-lived representations are dedicated to action and require a strict temporal and spatial correspondence between the goal to be reach and the response directed at it. 'Perceptual', long-lasting representations do not require such a stimulus-response correspondence. These 'intermediate' representations can be memorized and give rise to delayed motor responses, or to responses at a distance from the stimulus (e.g. in a different plane), or to an explicit verbal response on the perceived localization of the stimulus.

From these hypotheses, one can predict that optic ataxia would be improved when patients are required to perform either delayed or displaced responses. The present study was aimed at checking this point. We have compared the visuomotor performance of a 30 year-old patient (IG) with a severe optic ataxia (bilateral parietal infarction) to that of a normal age-matched control subject (HM). Ss were required to point at 5 visual targets (LED) turned on separately in total darkness. Pointing responses with the right hand were recorded by using an optoelectronic motion analyzer (Optotrak), in 3 main conditions: 'direct' pointing (goal-directed movement), 'indirect' pointing with either a delay of $5 \mathrm{sec}$ between stimulus offset and movement onset, or a spatial separation between stimulus (at eye level) and response (40 cm below, on the pointing table). In the 'direct' open loop condition, IG showed a systematic leftward bias (consistent with her more severe deficit on the right hand), with a mean final constant error of $5-6^{\circ}$ for each of the 5 visual targets, as well as a leftward shift of the initial direction of the hand trajectory. In the delayed response condition, in contrast with more scattered final errors observed in the control subject HM, IG's responses did not degrade compared to the 'direct' pointing condition. Final errors tended to decrease (although not significantly), and initial trajectory direction improved more consistently. In the displaced response condition, even more contrasting with HM's performance, mean constant error as well as initial direction improved dramatically in IG. These results indicate that optic ataxia patients, although lacking a mechanism of direct visuomotor transformation for visually elicited hand actions, still have access to 'intermediate' spatial representations, allowing them to indicate deliberately with the hand the perceived position of visual targets.

\section{DP8}

EXTRARETINAL INFLUENCES ON SPATIAL PERCEPTION: EFFECTS OF LATERAL HEAD TILT ON THE SUBJECTIVE VERTICAL AND HORIZONTAL IN BRAIN LESIONED SUBJECTS

G. Kerkhoff

EKN, Dept. Neuropsychology, City Hospital Bogenhausen, Dachauerstr. 164, D-80992 Munich, Germany

Many studies have documented extraretinal influences (eye-/head-position) on neuronal activity in animals as well as on spatial perception in patients with unilateral brain lesions $(1,3)$. Likewise, head-on- 
trunk position modulates the perceived direction of midline in patients with hemineglect after right parietotemporal lesions (2). In two recent studies $(4,5)$ we showed that right temporo-parietal lesions impair the subjective judgment of vertical, horizontal or oblique orientations in the frontal plane, both visually and tactually. Here we investigated how lateral head-tilt (head upright $=0$-tilt, $25^{\circ}$-clockwise tilt, $25^{\circ}$-counterclockwise tilt) affects the perception of the vertical/horizontal in the visual and tactile modality. Constant errors were obtained in the four tasks using special devices $(4,5)$. Starting position of the stimulus and experimental conditions were balanced. Visual tests were performed in total darkness, tactile tests with subjects blindfolded. Trunk position was always upright and stabilized using an experimental chair. Head orientation was modulated with a tiltable head- and chinrest. Five normal subjects, six right brain damaged subjects (RBD, temporo-parietal lesions) with a disturbance of the static visual vertical, and six left-brain damaged subjects (LBD, temporo-parietal lesions) without a deficit of the visual vertical were examined. The results show that RBD-subjects performed significantly worse in all tasks as compared to both other groups thus replicating our previous results of a counterclockwise tilt of the visual and tactile vertical/horizontal in rightsided parietotemporal lesions $(4,5)$. Counterclockwise head-tilt aggravated this deficit considerably, while clockwise head-tilt improved it. Only minor effects of head orientation were observed in the two other groups, both compensating easily for the head-tilt (constant errors $<2^{\circ}$ ). In conclusion, 1) lateral head-on-trunk orientation exerts a dramatic influence on spatial perception of verticality and horizontality in RBDpatients with parieto-temporal lesions, irrespective of the modality tested. Only small effects of this manipulation were observed in LBD and normal subjects. 2) This instability of spatial perception is likely to contribute to the postural problems of these patients, since their judgment of verticality varies with rather small changes in head position. 3) Hence, the head-vertical axis plays a similar important role in determining spatial orientation in the frontal plane as the head- and trunk-sagittal-axis for determining straight ahead or the midline in space. Both processes appear to be disrupted in right parieto-temporal lesions.

(1) Andersen, R.A. et al. (1997) Ann. Rev. Neurosci., 20:303; (2) Schindler, I. \& Kerkhoff, G. (1997) NeuroReport 8:2681; (3) Bremmer, F. et al. (1998) Eur. J. Neurosci., 10:153; (4) Kerkhoff, G. \& Zoelch, C. (1998) Exp. Brain Res., 122:108; (5) Kerkhoff, G. (1999) Neuropsychologia, in press.

\section{DP9}

HEAD DIRECTION AND OPTOKINETIC STIMULATION MODULATE SPACE BASED BUT NOT WORD-BASED NEGLECT DYSLEXIA

G. Kerkhoff ${ }^{*}$, G. Kriz ${ }^{1}$, I. Keller ${ }^{2}$, and C. Marquardt ${ }^{1}$

'EKN, Dept. Neuropsychology, Hospital Bogenhausen, Dachauerstr. 164, D-80992 Munich, Germany and ${ }^{2}$ Neurological Clinic Bad Aibling, Germany

Visual attention may operate both space- and object-based. Consequently, patients with parietotemporal lesions show space-based and/or object-centered neglect. Space-based neglect is modulated, among other 
factors, by head-direction (HD) and large-field optokinetic stimulation (OKS). Furthermore, HD contributes significantly to space coding in animals. Space- and object-based attentional processes are required during text reading. Consequently, neglect patients may show impairments in both processes (space-based vs. word-based neglect dyslexia). An open question is the relationship of these both types of deficits in neglect. Here, we tested whether both mechanisms are dissociable by evaluating the effects of HD and OKS on spce and word-based neglect dyslexia. Nine patients with severe neglect dyslexia as determined by screening tests, ten right-brain-damaged control patients (RBD-controls) without neglect and eight normal subjects were tested under 6 experimental conditions: 1) Baseline, no OKS, head straight; 2) Head to the left (20), no OKS; 3) Head right, no OKS; 4) Head straight, OKS to the left (7.5 (s-1); 5) Head straight, rightsided OKS; 6) Baseline, no OKS, head straight. Indented texts (80 words) were displayed within a $20 \times 10 \mathrm{~cm}$ window on a computer screen. The head was fixed with a head/chinrest or stabilized by a second experimenter. Readings were tape-recorded and analyzed offline for space-based errors (omissions) and word-based errors (substitutions or partial modifications of a word/syllable). During OKS 100 squares drifted outside the window either to the left or right side. The following results were obtained. 1) Neglect patients showed significantly more space-based and wordbased reading errors than the two other subjects groups. They had $26 \%$ overall reading errors; $88.5 \%$ of these were space-based errors, $11.5 \%$ were word-based errors. 2) Contralesional, leftward HD significantly reduced space-based errors, while rightsided HD had no significant effect as compared to the two baselines. 3) Leftward OKS significantly reduced space-based reading errors, while rightsided OKS led to identical results as in the two baselines. 4) Neither OKS nor HD influenced word-based neglect errors significantly. 5) No effects of HD or OKS were obtained in the RBD-control and normal subjects who performed nearly errorless $(<1 \%$ errors). In conclusion, $\mathrm{HD}$ and OKS both exert a powerful influence on space-based neglect dyslexia but do not affect word- or object-based neglectdyslexia, at least not within the chosen experimental setup. This indicates the dissociability of the underlying attentional processes 2) Both manouevers (HD, OKS) may be used for the rehabilitation of space-based neglect dyslexia while other therapeutic techniques have to be developed for object centered neglect dyslexia.

\section{DP10}

\section{THE INFLUENCE OF DISTANCE AND MOTOR RESPONSE ON VISUAL HEMINEGLECT}

I. Keller ${ }^{1}$ I. Schindler ${ }^{2}$, and D. Golz ${ }^{3}$

${ }^{1}$ Neurological Clinic Bad Aibling, ${ }^{2}$ EKN-Clinical Neuropsychology Research Group, Munich, and ${ }^{3}$ Institute of Psychology Friedrich-Wilhelm-University Bonn, Germany

Several reports indicate a dissociation of attentional systems for near and far space in patients with visuospatial neglect $(1,5,6)$. There is also good evidence from neurophysiological studies that activation of different motor-systems in near and far space might be responsible for this effect $(2,3,4)$. We therefore investigated the influence of different response modes (verbal response vs motor response) on a horizontal 
line bisection task as well as a cancellation task in near and far space. Ten patients with left-sided visuospatial neglect were tested. Line bisection was realized by presenting a horizontal bar of $20 \times 1$ degree visual angle. The cancellation task consisted of a matrix with 36 digits and letters embedded in 30 randomly distributed distractors. Length and width of the matrix was $28 \times 21$ degrees. In near space, stimuli were presented at a distance of $60 \mathrm{~cm}$ on a computer screen. In far space, stimuli were projected with a colour TFT overhead display at a distance of $160 \mathrm{~cm}$. During the verbal response mode, the patient adjusted a movable slit to the subjective midline position by verbal command. During the motor response mode the patient had to point to the midline position with either a pencil or a pointer (far space). In the cancellation task the patient named or pointed to all letters and digits he could see. Line bisection: In the verbal response mode five patients showed an increase of abberant rightwards bisection from near to far space. In the motor response mode only one patient shifted the midline to the right side whereas four patients committed a leftwards shifting of bisection. The angular error was greater with motor response in near (nine patients) and far space (five patients). Cancellation task: Eight patients displayed more than $50 \%$ omissions. With the exception of one patient with damage to the corpus callosum, none of the remaining nine patients showed any effect of distance or response mode. The more prominent neglect with line bisection in far space is in agreement with previous findings (5). The interaction between distance and response mode might be due to different motor programmes used for near and far space as proposed by Cowey et al. (6). Performance in the cancellation task was nearly constant for all four experimental conditions indicating that line bisection and visual search may represent independent tasks also executed by different brain systems.

(1) Halligan \& Marshall (1991), Nature, 350:498; (2) Holand et al. (1980) J. Neurophysiol., 43:118; (3) Roland et al. (1980) J. Neurophysiol., 43:137; (4) Rizzolatti et al. (1983) Brain, 106:655; (5) Cowey, et al. (1994), Neuropsychologia, 32:1059; (6) Cowey, et al. (1999). Neuropsychologia, 37:12.

\section{DP11}

RIGHTWARD SIZE UNDER ESTIMATION IN A PATIENT WITH RIGHT VISUOSPATIAL NEGLECT

C.L. Pritchard ${ }^{1}$, H.C. Dijkerman ${ }^{l}$, A.D. Milner ${ }^{1}$, and R.C. Roberts ${ }^{2}$

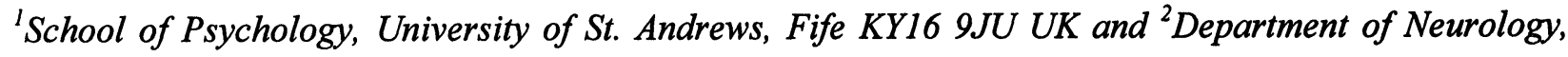
Ninewells Hospital, Dundee, UK

We report a left-handed patient (DL) who sustained a large left hemisphere lesion as the result of an occlusion of the middle cerebral artery. DL showed no signs of aphasia or ideomotor apraxia, symptoms typically associated with left brain damage. However, he did have severe and persistent right visuospatial neglect. This suggests that DL is an example of an individual with 'atypical' or 'reversed' cerebral dominance. When bisecting lines, DL made large leftward errors that were reduced by cueing him to the right end of the line and increased by presenting the lines in right space. DL was also presented with prebisected lines (The Landmark Task) and was asked to indicate which half of the line appeared to him to be shorter. When lines were bisected centrally, and when they were bisected up to $5 \mathrm{~mm}$ to the left of 
centre, DL consistently indicated that the right half of the line appeared shorter. This rightward size underestimation was also evident in a visual perceptual size judgement task. DL underestimated the size of horizontal lines, horizontal rectangles and circles presented to the right of his midline relative to those presented to the left. However, this was not the case for radial lines. We additionally assessed DL's ability to make size judgements based on tactile rather than visual information. Again, the size of objects presented on the right was underestimated as compared to those placed on the left.

Thus, DL demonstrated a right size underestimation similar to the leftward size underestimation previously reported in right brain damaged patients with left neglect. Previous cases of right neglect following left hemisphere lesions have been reported: however, this is the first time that such a patient has been examined systematically for a possible 'reversed' distortion in size perception.

DP12

THE NATURE OF HYPERATTENTION IN THE IPSILESIONAL VISUAL HEMIFIELD OF NEGLECT PATIENTS

E. Natale ${ }^{\prime}$, C. Cavina-Pratesi ${ }^{l}$, A. Maravita ${ }^{I}$, P. Manganotti ${ }^{l}$, L. Posteraro ${ }^{2}$, N. Smania ${ }^{3}$, and C.A. Marzi ${ }^{l}$

${ }^{I}$ Dept. of Neurological \& Visual Sciences, Univ. of Verona, Italy, and ${ }^{2}$ Rehabilitation Unit USL 20 Viadana, Mantova, ${ }^{3}$ Rehabilitation Unit, Policlinico Borgo Roma, Verona, Italy

Hemineglect consists in a profound unawareness of the contralesional hemispace (visual, tactile or auditory) which occurs in patients with right parietal lobe damage. In a previous study (Smania et al., Brain, 1998) we found that neglect patients when tested on a simple visual reaction time (RT) paradigm with brief flashes lateralized either to the contralesional or to the ipsilesional hemifield, show dramatically opposite results. In the former hemifield, overall detection rate is poor and worsens as one goes from central to more peripheral eccentricities of stimulus presentation; the same is true for RT which becomes progressively slower at higher eccentricities. In contrast, in the latter hemifield, both accuracy of detection and RT show a paradoxical improvement as one goes from central to more peripheral visual field location. We called this effect "hyperattention" by analogy with other behavioural phenomena classically described in neglect patients following ipsilesional sensory stimulation. In the present study we tried to ascertain what kind of attention is hyperattention by manipulating the subjects' expectation as to the location of the incoming stimulus. We studied 8 neglect patients and 9 age-matched controls. There were three modalities of stimulus presentation: in the first, the position of the stimuli in either contra- or ipsilesional hemifield was the same for each block of trials; in the second, the position was randomised both across hemifields and across positions and, finally, in the third condition, hemifield was blocked while stimulus location was randomised. The results were statistically analysed only for the ipsilesional hemifield because overall detection rate in the contralesional field of neglect patients was poor. However, it is important to mention that their overall performance improved in the blocked position condition and this shows that neglect patients benefit from allocating attentional resources to a specified 
location in the neglected side. In the ipsilesional hemifield there was an overall performance improvement of neglect patients when tested under blocked position conditions but, as in control subjects, there was no hyperattention effect, i.e., RT slowed down from central to more peripheral positions. On the contrary, a paradoxical speeding up at higher eccentricities was present both under completely randomised conditions and under blocked hemifield conditions. These results show that hyperattention is related to an exaggerated ipsilesional shift of attention that is present when stimulus location is uncertain and therefore is likely to concern automatic rather than voluntary allocation of attention.

\section{DP13}

SPATIAL POSITIONS OF THE LEFT HAND INFLUENCE SOMATIC NEGLECT BUT NOT DISOWNERSHIP OF THE HAND IN A RIGHT BRAIN-DAMAGED PATIENT.

M. Zampini ${ }^{l}{ }^{*}, V$. Moro $^{2}$, F. Poletto ${ }^{3}$, and S. Aglioti ${ }^{4}$

${ }^{I}$ Dept. of Neurological and Vision Sciences, Physiology Section, Univ. of Verona, ${ }^{2}$ Ospedale "Sacro

Cuore", Negrar, ${ }^{3}$ Centro Riabilitazione, Ospedale Chiarenzi Zevio, and ${ }^{4}$ Department of Psychology,

University of Rome, "La Sapienza", Italy

Somatic delusions like supernumerary phantom limb and denial of ownership of a given body part have been reported following damage to the right side of the brain. These symptoms typically co-occur with personal or extrapersonal neglect and anosognosia. A right brain-damaged patient has been reported in whom a cold left ear caloric stimulation of the contralesional ear brought about an improvement not only of extrapersonal neglect but also of the disownership of her left hand. This result suggests that these symptoms may rely upon common neural substrates. Here we report a 63 year-old woman who presented with florid left personal and extrapersonal neglect following a right hemisphere stroke. In spite of the fact that the patient was alert and co-operative she vehemently denied that her plegic left hand were her own. We tested whether or not changing the spatial position of the disowned hand influenced somatic neglect and the capability to attribute the ownership of different parts of her body. Three blocks of 10 single ( 5 left and 5 right) and 10 double touches were delivered to the dorsal surface of the fingers. Each block differed for the position of the left hand that could be at $0^{0}$ or $70^{\circ}$ in the left or the right hemispace. In each trial, the patient was also asked to rank ownership of the finger stimulated by using a 7-point scale which ranged from 1 (resolute denial of ownership) to 7 (correct attribution of ownership). No positionrelated changes in denial of ownership were detected. In logical contrast with the denial of ownership the patient reported some of the stimuli delivered to her left hand. Remarkably, correct detections were $0 \%$, $10 \%$, and $100 \%$ when the left hand was in left, central or right position respectively. Thus, in this patient, manipulations of the spatial position of the hand reduce somatic neglect but leave unaffected denial of ownership of the contralesional hand. This dissociation suggests at least partially separate neural substrates for representing hand in space and attibuting ownership of body parts. 


\section{DP14}

NECK MUSCLE VIBRATION AND VISUAL EXPLORATION TRAINING: VISUAL AND CROSSMODAL EFFECTS ON NEGLECT REHABILITATION

I. Schindler ${ }^{\prime *}$, G. Kerkhoff', H.O. Karnath ${ }^{2}$, I. Keller ${ }^{3}$, and G. Goldenberg ${ }^{1}$

'EKN-Clinical Neuropsychology Research Group, Department of Neuropsychology, City Hospital

Bogenhausen, Dachauer Str. 164, D-80992 Munich, Germany, ${ }^{2}$ Department of Neurology, University of Tübingen, Germany, and ${ }^{3}$ Neurological Rehabilitation Center, Bad Aibling, Germany

Recent studies revealed an improvement of visual exploration in neglect during vibration of the contralesional neck muscles. It has been suggested, that vibration leads to a compensation of a supramodally distorted egocentric coordinate system. The aim of the present study was to investigate the therapeutic potentials of vibration e.g. with respect to the transfer in different visual tasks and modalities. 20 patients with neglect were subjected to a cross-over design: In the first treatment phase 10 patients received 15 sessions of specific visual exploration training. In the second phase (15 sessions) this training was combined with neck muscle vibration. The other half of the patients received both treatments in the reverse order. Performance was measured for six times: Three baseline measurements across three weeks before treatment onset, two posttreatment measurements and one follow-up after two months. The results revealed significant and stable improvements after visual exploration treatment in cancellation and text reading but not in the subjective straight ahead estimation and in a tactile exploration task. After the combination treatment a significant additive improvement was observed in these tasks. In contrast, none of the therapeutic methods led to an amelioration in size distortion in a visual size estimation task. The present data suggests a considerable additional potential for the improvement of neglect-related deficits by a combination of visual exploration training with neck muscle vibration. The specific advantage of this treatment seems to be a positive crossmodal treatment effect in patients where spatial reference systems working in egocentric coordinates are affected. The stable size distortion error is an indication of further disturbed visual-spatial subsystems in neglect which may be coded in an allocentric coordinate frame.

\section{DP15 \\ EXECUTION OF APRAXIC IMITATIONS AFTER CALLOSAL DISCONNECTION J. Hermsdörfer*, K. Laimgruber, and G. Goldenberg EKN Clinical Neuropsychology Research Group, Hospital München-Bogenhausen, Germany}

We report the case of a patient with callosal disconnection due to repeated bleedings and operations which completely destroyed the middle and posterior parts of the corpus callosum. On clinical examination, apraxia of the left hand was confined to meaningful movements on verbal command. The imitation of meaningless gestures, which required arm movements with different final positions of the hand in the relation to the head, was normal on both hands. If, however, the same task was tested tachistoscopically, he was correct only if the required gesture was flashed into his right visual field (VF) 
and he performed with the right hand (19 of 20 correct); all other visual-field/hand combinations yielded many errors (6-10 correct). To study whether movement execution reflects the same performance pattern, the kinematics of arm/hand movements during the imitation of meaningless gestures were evaluated under the different experimental conditions using an ultrasonic motion analysis system (CMS 70, Zebris, Isny, Germany). Under the standard condition with normal vision of the demonstrated gesture, movement kinematics exhibited the invariant features of skilled preprogrammed movements previously described in control subjects. Various movement parameters such as maximum acceleration, maximum velocity, number of velocity peaks and adjustment path length were similar under the full vision and rightVF/right-hand combination, but deteriorated under the other combinations. Thus, on the average, the quality of movement execution reflected the frequency of apraxic errors. Closer analysis of the performance under the error provoking combinations yielded mainly three distinguishable patterns: Movements that ended in incorrect final positions were typically executed with defective kinematics, correct final positions were sometimes associated with abnormal but more frequently with normal kinematics. In the latter case, kinematics fully corresponded to those of the favourable conditions. Thus, only the left hemisphere was able to consistently build up an adequate representation of the demonstrated meaningless hand gestures, and to precisely and smoothly execute them with the contralateral right hand under tachistoscopic conditions. Movement kinematics may reflect the accuracy and precision of the representations: If the representation is precise, the kinematics of skilled preprogrammed movements can be implemented. Each hemisphere is in principal able to subserve this programming. However, if the representation is vague or inaccurate, kinematics deteriorate. In some trials prolonged execution and corrective movements may nevertheless result in correct final positions.

\section{DP16}

\section{INTER-HEMISPHERIC DELAY FOR SIMPLE VISUO-MOTOR RESPONSES IN A PATIENTS WITH A LESION OF THE BODY OF THE CORPUS CALLOSUM}

L.G. Grammaldo ${ }^{*}$, F. Tomaiuolo', U. Nocentini', and C. Caltagirone ${ }^{2}$

'I.R.C.C.S. 'Santa Lucia', Rome, Italy, and ${ }^{2}$ Università di Tor Vergata, Rome, Italy

When simple visual stimuli are presented to the hemifield contralateral to the responding hand (crossed condition) slower reaction times (RTs) are observed than when stimuli are presented to the ipsilateral hemifield (uncrossed condition). The crossed uncrossed difference in RTs (CUD) is of about 3 ms (see Marzi et al. 1991 for a review). It has been hypothesized that this difference is due to the transfer of visual signal from one hemisphere to the other, ostensibly via corpus callosum (CC). A much longer CUD is observed in subjects with agenesia of CC (about $20 \mathrm{~ms}$ ) and in patients with complete callosotomy (about $60 \mathrm{~ms}$ ). It is under debate of which part of the corpus callosum is normally involved in speeded crossed visuo-motor RTs. Recently, Tassinari et al. (1994) investigated 7 patients who had a surgical lesion of the anterior portion of the CC and 1 patient who had an agenetic absence of the posterior third of the $\mathrm{CC}$ including the splenium. Both the patients with the lack of the anterior section of 
the CC and the one with the lack of the splenium of the CC had a CUD in within the range of normal human. However, Di Stefano et al (1992), Iacoboni et al. (1994) and Geschwind et al. (1995) did find patients with partial callosotomy (sparing the spleniun of the CC) who showed a CUD similar to those observed in patients with complete absence of CC. We studied a 65 years patient (V.P.) who suffered from an ischemic vascular lesion of the $\mathrm{CC}$ extending from the genu to the most caudal part of the body and sparing the splenium. The CUD was measured using a simple reaction time paradigm with unimanual response to lateralized flashes at $6^{\circ}$ and $10^{\circ}$ of eccentricity. V.P. showed an overall CUD of about $40 \mathrm{~ms}$. These results suggest that the callosal transfer of simple visuo-motor information is related to axons positioned into the body of the CC.

\section{DP17}

EXECUTION AND MENTAL REHEARSAL TIME COUPLING IN DIFFERENT MOTOR TASK IN NORMAL SUBJECT AND PATIENTS WITH CEREBELLAR DAMAGE

V.Filippini $^{l *}$, M.G. Leggio ${ }^{l, 2}$, M. Capecci ${ }^{l, 4}$, L. Petrosini ${ }^{l, 2}$, and M. Molinari ${ }^{l, 3}$.

'IRCCS S. Lucia, ${ }^{2}$ Dept. of Psychology, University of Rome "La Sapienza", Rome, ${ }^{3}$ Institute of Neurology, Catholic University, Rome, Italy, ${ }^{4}$ Neurorehabilitation Clinic, University of Ancona, Italy

To analyze brain mechanisms underlying mental representation of actions, the present study investigates the role of the cerebellum in affecting the timing of both execution and mental rehearsal of motor acts. Cerebellar patients affected by degenerative or focal pathologies and age and education matched controls were tested. Four tasks were performed: 1) a continuos thumb-fingers opposition sequence paced by a metronome; 2) four pairs of hand postures; 3) moving the tip of a hand-held stylus; 4) a complex purposeful movement sequence. Each task was repeated 3 times either mentally or actually always starting with the mental performance. For each subject both right and left sides were studied. Normal subjects did not show significant differences between execution and rehearsal times with the notable exception of task 1 . In this task they displayed execution performance significantly faster than mental rehearsal. All patients presented longer times than controls for both actual execution and mental rehearsal in all tasks. In tasks 3 and 4 time coupling between execution and mental rehearsal is not preserved for degenerative lesion patients and only for the affected side in patients with focal lesions. The present data indicate that: 1) execution and mental time coupling depends on the characteristics of different motor task in normal subjects as well as in patients with cerebellar damage; 2) cerebellar lesions affect both actual and mental movements times demonstrating thus that the cerebellum is among the cerebral structures involved in both execution and mental times. 


\title{
DP18
}

\section{VISUAL EVENT RELATED BRAIN POTENTIALS IN DYSLEXIC CHILDREN}

\author{
A. Shankardass*, R. I. Nicolson, A. J. Fawcett
}

Dept. Psychology, University of Sheffield, Sheffield S10 2TP, UK

It is now well established that dyslexic children show performance deficits in a wide range of tasks at the behavioural level as well as neurophysiological deficits at the systems level. Recent evidence has indicated a difficulty in the speed of processing of most stimuli, which is now thought to play a contributory role towards most dyslexic difficulties. The purpose of this study was to assess the nature, extent and loci of this temporal processing deficit in the visual domain in an omission choice reaction task using new event related brain potential (ERP) techniques. It replicates and extends a previous study (Fawcett et al., 1992) in the auditory domain that used older, established ERP techniques. The first cohort of six 16 year old dyslexic children and their age and IQ matched controls were tested on a visual target detection task using the oddball paradigm, in which they were required to respond only to rarely occurring, relevant stimuli (target) and ignore frequently occurring, irrelevant stimuli (non-target). While the task was administered, their visual ERPs were recorded using a high density electrode geodesic sensor net. A comparison was made of the P300 component of the ERP waveform (representing termination of stimulus classification) from the $\mathrm{Cz}$ (central midline) location on the scalp, referenced to the linked mastoids. It was found that the P300 latency to peak for the rare target stimuli was significantly longer for the dyslexic children than for the controls (mean difference $34.67 \mathrm{msec}, \mathrm{z}=-1.92$, $\mathrm{p}=0.05$ ). Hence the study was consistent with the earlier findings in a different modality and provided further evidence that this deficit in speed of processing in dyslexic children is evident even for non linguistic visual stimuli and appears to be linked to stimulus categorisation. Further analysis of the amplitudes and latencies to peak of various components of the ERP waveform as well as their scalp distributions will be presented for the above and further cohort of subjects. The greater spatial resolution of the high density electrode array should facilitate novel interpretation of the data in terms of anomalies in time course of activation across different regions of the brain in dyslexics and help elucidate the brain structures contributing to this deficit in their speed of processing.

\section{DP19}

MAGNOCELLULAR DEFICIT IN DYSLEXIA: EVIDENCE FROM CONTRAST SENSITIVITY TEST

A. Grabowska* and D. Bednarek

Nencki Institute of Experimental Biology, Dept. of Neurophysiology, 3 Pasteur St., 02-093 Warsaw, Poland

It has been proposed that dyslexia may result from a deficit to the magnocellular channel of the visual pathway. The purpose of the present study was to verify that hypothesis by measuring contrast sensitivity to stationary and moving gratings of various spatial frequencies. Fourteen control and 14 dyslexic children 
participated in the study. They were matched in IQ and age ( 9 years old). The reading and writing abilities of dyslexics were substantially ( 2 years) behind their chronological age. Contrast sensitivity was measured with VSG2 visual generator. Sine-wave, horizontal, black-and-white gratings of $0.5 ; 1.5 ; 3.8 ; 8 \mathrm{c} / \mathrm{deg}$ spatial frequencies were presented on a computer high resolution screen. On every second trial the gratings were displayed at high contrast and the subjects decreased it (by pressing a lever) until they could no longer see the stimuli; and on every second trial the stimuli were displayed at so low contrast that the subjects were not able to see them. On those trials subject's task was to increase the contrast until they could just detect the stimuli. Based on such estimations a computer program established the contrast threshold for each spatial frequency. Five different conditions of stimuli presentation were used: 1) stationary gratings;

2) grating reversals of $2 \mathrm{c} / \mathrm{sec}$ temporal frequency; 3) grating reversals of $8 \mathrm{c} / \mathrm{sec}$ temporal frequency; 4) gratings drifting across screen with a velocity of $2 \mathrm{c} / \mathrm{sec} ; 5)$ gratings drifting with a velocity of $8 \mathrm{c} / \mathrm{sec}$. The results showed that dyslexics were more sensitive to contrast than controls. However, the pattern of the contrast sensitivity function in the two groups depended very much on the experimental condition. In agreement with earlier findings, in the control group introduction of movement increased contrast sensitivity to low spatial frequency gratings whereas it had a decremental effect on high spatial frequency gratings. Contrary to that, dyslexics showed lower contrast sensitivity to moving stimuli than to stationary ones for all spatial frequencies. This suggests that dyslexic children differed from controls specifically in condition when magnocellular channel was activated (low spatial frequency, moving gratings). The study supports the view that dyslexia may result from a deficient functioning of the magnocellular channel. At the same time it provides a promising method for early detection of a visual disorder that may cause dyslexia.

\section{DP20}

\section{A NON-LINGUISTIC EXPRESSION OF AGRAMMATIC APHASIA}

T. Lelekov ${ }^{l}$, P.F. Dominey ${ }^{l}$, C. Chosson-Tiraboschi ${ }^{l}$, J. Ventre-Dominey $^{2}$, D. Labourel ${ }^{3}$, F. Michel ${ }^{1,3}$, and B. Croisile ${ }^{1,3}$

${ }^{l}$ Institut des Sciences Cognitives UPR - 9075 - CNRS, 69675 Bron, France , ${ }^{2}$ Vision et Motricité, Unité 94 INSERM, 69500 BRON, France, and ${ }^{3}$ Laboratoire de Neuropsychologie, Hôpital Neurologique, 69003 Lyon, France

Individual words and morphemes carry their own sense, but it is their organization into grammatical sentences that provides the true richness for the expression of meaning. Left hemisphere lesions in and around the perisylvian cortex including Broca's area can lead to agrammatic aphasia, an impaired ability to exploit this syntactic structure for sentence comprehension. Agrammatic aphasics understand sentences whose thematic roles follow the "canonical" order (Agent - Object - Recipient) like 'John gave the ball to Mary'. They fail, however, to understand non-canonical sentences like 'The ball was given to Mary by John'. We test the hypothesis that this deficit results from an impairment in performing serial order transformations for non-canonical orderings, and that this impairment is not restricted to natural language. We test this hypothesis in a total of 9 aphasic patients in two experiments. To test non- 
linguistic sequence processing we introduce the following distinctions: The sequences $\mathrm{ABCBAC}$ and DEFEDF have different surface structures, but share abstract structure 123213, and are thus isomorphic sequences. In this abstract structure, the second triplet (213) is a transformation of the first (123), and is thus considered to be non-canonical, while abstract structure 123123 is canonical. In Experiment 1, we demonstrate in a serial reaction time task that, like age matched control subjects, agrammatic patients can learn the serial order of sensorimotor sequences. They fail however to learn the non-canonical abstract structure that can transfer to new, isomorphic sequences. In Experiment 2, we demonstrate that both in natural language comprehension, and letter-sequence discrimination tasks, agrammatic patients correctly process canonical but not non-canonical structure. Most important, there is a significant correlation between impairment in the linguistic and non-linguistic tasks $\left(r^{2}=0.86, p=0.003\right)$. In addition, we observe that in both the linguistic and non-linguistic tasks, there is a significant performance impairment for non-canonical vs. canonical orders (Structure effect: $p<0.005$ ), and no influence of the Task (linguistic vs. non-linguistic) on this effect (Structure $x$ Task interaction: $p=0.8$ ). These data support the hypothesis that agrammatic aphasia is related to an impaired capacity to process non-canonical order in linguistic as well as non-linguistic sequences. The correlation between performance in the linguistic and non-linguistic tasks suggests a common underlying basis, a possibility that remains to be explored.

\section{DP21}

UNIQUE CASE OF SOTOS SYNDROME IN AN IDENTICAL TWIN: NEUROPSYCHOLOGICAL FINDINGS OF A THREE YEAR FOLLOW-UP

J.M.W.R Berndsen-Peeters ${ }^{l}{ }^{*}$, H. Van der Vlugt ${ }^{l}$, L.M.G. Curfs ${ }^{2}$, and J.-P. Fryns ${ }^{3}$

${ }^{I}$ Tilburg University, Tilburg, The Netherlands, ${ }^{2}$ Center of Clinical Genetics, Maastricht University, The Netherlands, and ${ }^{3}$ Center of Human Genetics, Leuven University, Belgium

An identical female twin, born June 17, 1988 was diagnosed suffering form Sotos syndrome four years ago. A full-scale Neuropsychological Assessment was done three times with an interval of 15 and 14 months. Data were compared between the twins and over time. The typical characteristics are highlighted and will be discussed in terms of a possible connection with the NLD-syndrome or as a clear cut aspects and characteristics of the Sotos syndrome itself. Results will also be compared with the very few neuropsychological data available in the literature today. 
DP22

ISOLATED AMNESIA OF INSIDIOUS ONSET WITH DISPROPORTIONATE IMPAIRMENT OF RETROGRADE MEMORY : A CASE STUDY

A. Daniele ${ }^{l}$, P. Dialmi ${ }^{l}$, M.C. Silveri ${ }^{l}$, D. Perani ${ }^{2}$, and G. Gainotti $i^{l}$

${ }^{\prime}$ Institute of Neurology, Catholic University, Rome, Italy and ${ }^{2} I N B$ CNR and University of Milan, Scientific Institute San Raffaele, Milan, Italy

A case is reported of a 69-year-old patient who, at the age of 64 years, started to experience difficulties in remembering even meaningful recent autobiographical events. Such difficulties became progressively worse over the following two years, and remained fundamentally unchanged in the last three years. At the age of 62 , he had started to have partial complex seizures, characterised by visual sensation of light flashes associated with a feeling of well-being. When the patient was referred to us at the age of 66 , elementary neurological examination was unremarkable. Night sleep polygraphic recordings showed spike-and-wave discharges in the right temporal regions during non-REM sleep. A treatment with carbamazepine $(600 \mathrm{mg} / \mathrm{die})$ was started, which has been successful in preventing partial complex seizures. Magnetic resonance imaging (MRI) revealed mild diffuse cortical atrophy, mainly involving the frontal and parietal lobes. No signal abnormality was detected in the hippocampal formation, which were of normal size. A positron emission tomography (PET) study using $\mathrm{H}_{2}{ }^{15} \mathrm{O}$ showed a reduction of regional cerebral blood flow in the mesial frontal cortex bilaterally, and in the temporal poles, more markedly in the left pole. Neuropsychological examination revealed a disproportionate impairment of retrograde memory, with poor performance on tests of autobiographical memory for recent events, and on tests of memory for famous faces. Moreover, on tests of knowledge of famous events, the patient scored in the borderline area between pathological and normal performance. By contrast, his performance was in the normal range on most tests of anterograde memory, except for a task of visual episodic long-term memory assessing delayed recognition of pictures. Over a 3-year follow-up period, the patient performed with normal limits on various neuropsychological tasks assessing linguistic, praxic and "frontal" cognitive functions. We suggest that the co-occurrence in this patient of a 5-year history of isolated retrograde amnesia of insidious onset in association with a 7-year history of complex partial seizures could be due to a focal degenerative pathology. The possible role of anterior temporal lobe structures in deficits of retrograde memory is discussed.

\section{DP23}

MUSIC THERAPY IN BEHAVIOURAL DISTURBANCES DURING RECOVERY FROM PROLONGED POST-TRAUMATIC COMA

J.W. Weckel*, R. Formisano, F. Penta, V. Vinicola, M. Matteis, and S. Brunelli

I.R.C.C.S Santa Lucia, Rome, Italy

In the last years we investigated the efficacy of improvised Music Therapy on some post-traumatic 
behavioural disturbances such as psycho-motor agitation and/or inertia after severe traumatic brain injury. Music Therapy is based on the approach of Nordoff and Robbins (1977) and the work with comatose patients of Gustorff (1990). We examined $n=11$ patients with prolonged coma ( more than 30days), with Glasgow Coma Scale (GCS) $<8$ in the acute stage and Glasgow Outcome Scale (GOS) $<=3$ (severe disability). Absence of verbal initiative was the only criterion for the inclusion. Patients were examined on baseline according to GOS, Disability Rating Scale (DRS) (Rappaport, et al., 1982) and Coma Recovery Scale (CRS) (Giacino, et al., 1991) and post-coma scale (PCS) (Formisano et al., 1996). In particular we will describe patients with inertia and post-traumatic agitation during coma recovery with the aid of video recordings during the music therapy sessions. Music Therapeutic observations found out positive variations (video recordings) in 8 out off 11 patients, confirmed by a blind examiner in 5 patients out off the 11. While DRS and CRS failed to record the improvement of interactive abilities of the patients, PCS, which was also correlated with DRS and CRS in a previous study (Formasino et al., 1996) demonstrated a higher sensibility in evaluating significant changes of minimally conscious patients after severe traumatic brain injury especially with regard to post-traumatic psycho-motor agitation.

\section{DP24}

IDENTIFICATION AND DOSAGE OF PLASMATIC BONE GROWTH FACTORS IN TRAUMATIC BRAIN INJURY PATIENTS WITH HETEROTOPIC OSSIFICATION (H.O)

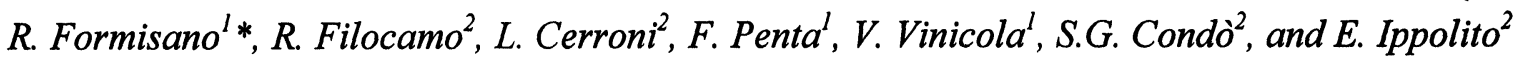

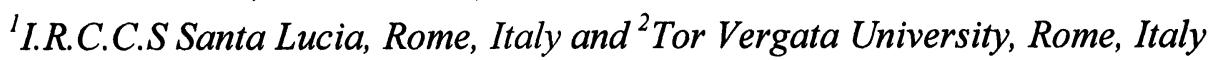

The traumatic lesions of the Central Nervous System (CNS) may induce, with unknown pathogenetic mechanisms, the development of heterotopic ossification (H.O), which cause often articular ankylosis, with important secondary rehabilitation problems. The frequency of $\mathrm{H} . \mathrm{O}$ has been widely described in traumatic brain injury and spinal cord injury patients (Garland, 1980, 1992; Sazbon, 1981; Stover, 1981). The radiographic evaluation of H.O is preceded by a plasmatic increase of the alkaline phosphatase (Furman, 1970), likely secondary to a possible osteogenic stimulation by a plasmatic factor produced by the trauma. In our study serum was taken from 27 patients with and without H.O suffering from severe traumatic brain injury and prolonged coma during rehabilitation and 10 healthy volunteers. Cell proliferation was measured after 24 hours using the cellular proliferation test (mitogen MTT-test). The activity of alkaline phosphatase (ALP) was measured by using p-nitrophenyl phosphate as the substrate. Eight sera have determined an increase of cell number respect to control and therefore showed significant mitogen activity. Nine sera out of all tested determined an increase of ALP production in osteoblasts respect to cells incubated with control sera. These results showed some interesting correlation with either the presence or the absence of H.O in the traumatic brain injury patients studied. 

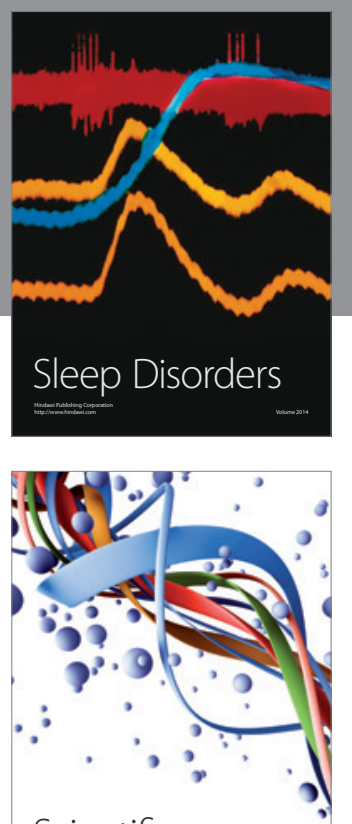

Scientifica
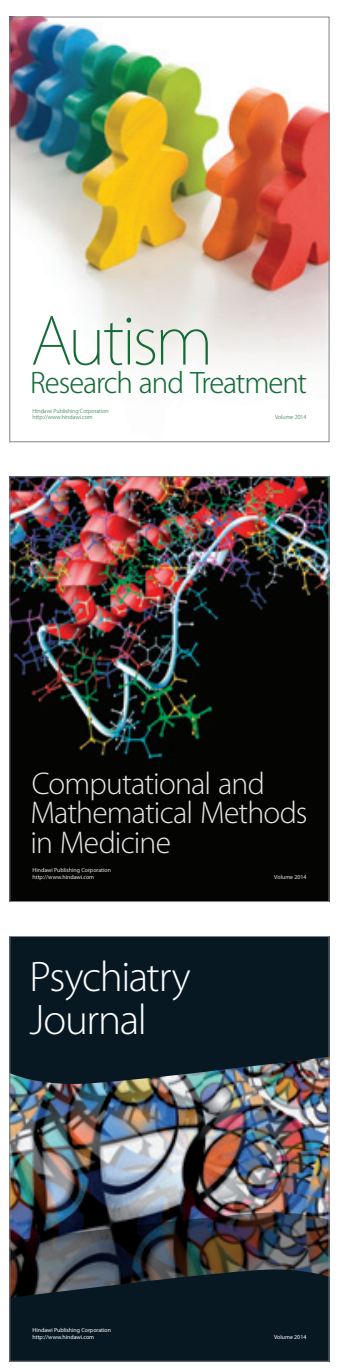
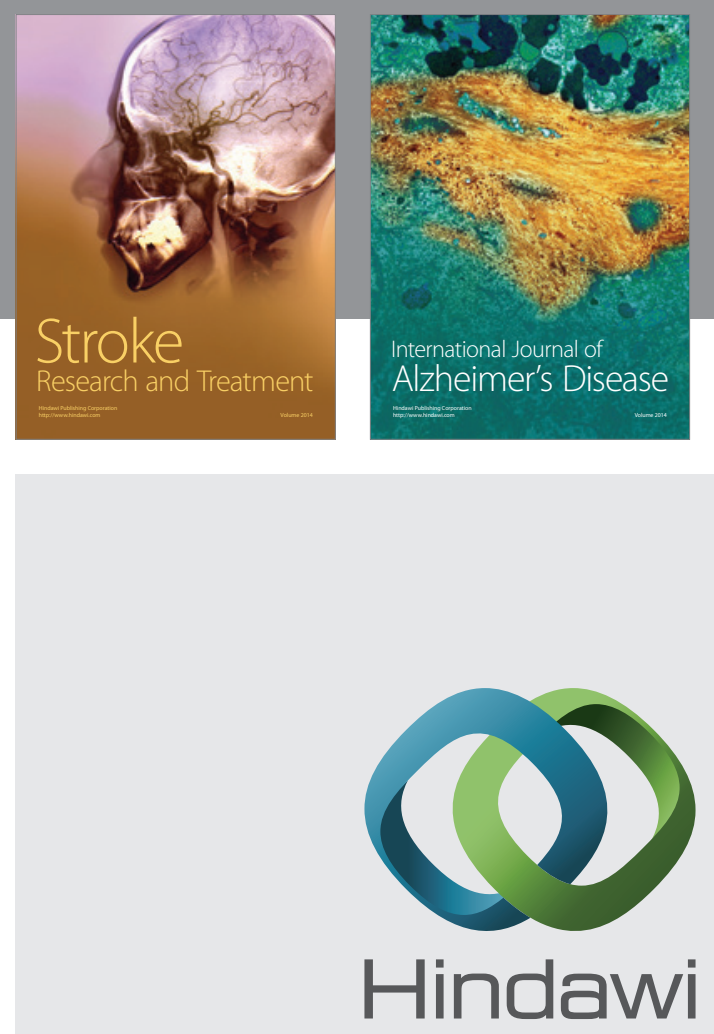

Submit your manuscripts at

http://www.hindawi.com
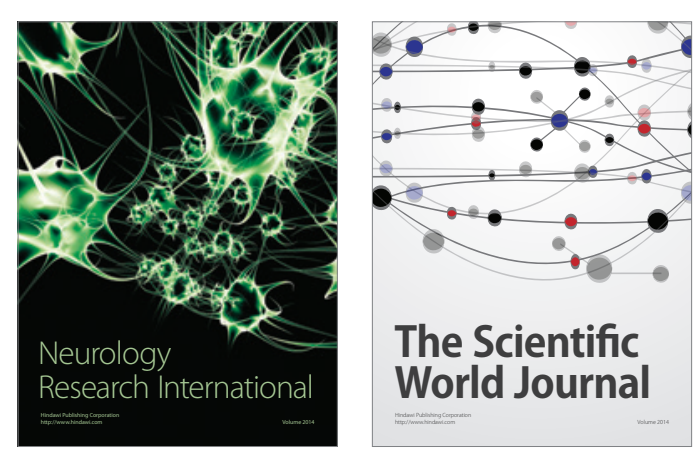

The Scientific World Journal

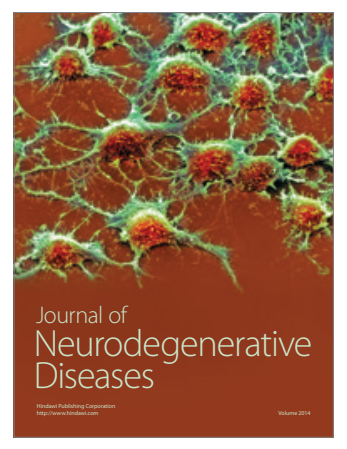

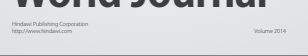

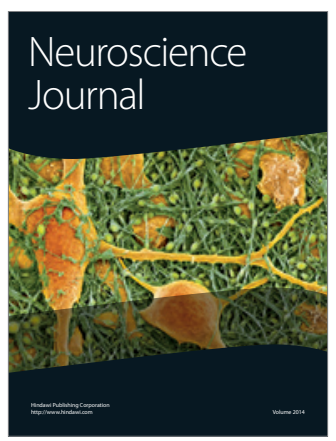

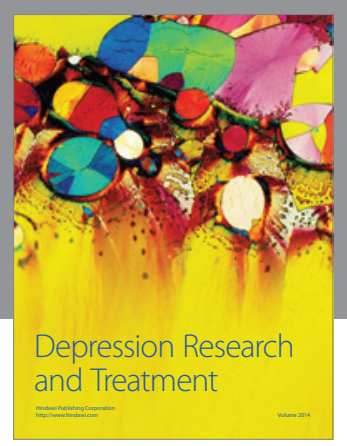
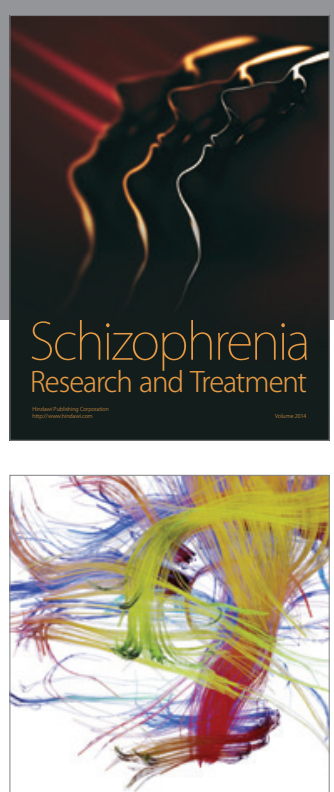

Brain Science

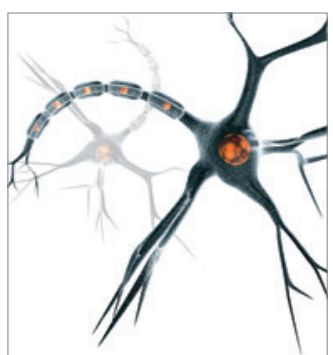

Neural Plasticity
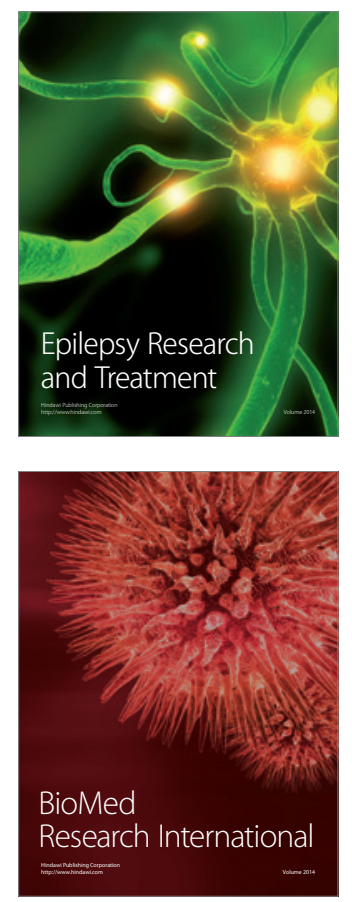

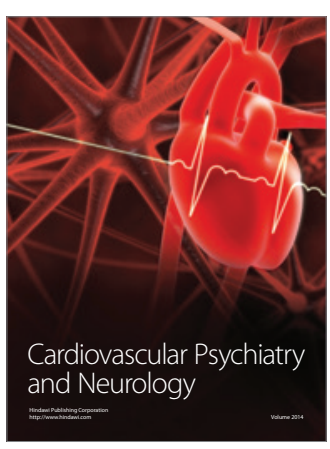

Parkinson's

Disease
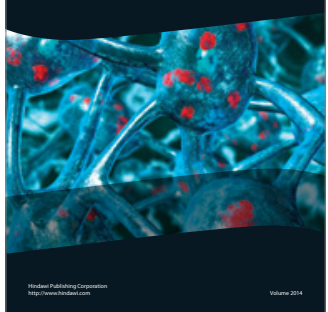